\title{
Strain rate dependence of dynamic flow stress considering viscous drag for 6061 aluminium alloy at high strain rates
}

\author{
Yuta CHIBA*, Kiyotaka SAKINO**
}

\begin{abstract}
In order to evaluate the strain rate dependence of the dynamic flow stress of aluminium alloys, 6061-O and -T6, high strain rate tests are performed at struin rates ranging from about $1000 / \mathrm{s}$ to $30000 / \mathrm{s}$, and strain rate reduction tests are also conducted in the strain rate range from about $10000 / \mathrm{s}$ to $20000 / \mathrm{s}$. A steep increase in the flow stress is observed for $6061-0$ at the strain rate of about $5000 / \mathrm{s}$. The above phenomenon, however, is not observed for 6061-T6 in the strain rate range where the strain rate reduction tests are conducted. A simplified model for a dislocation kinetics under a dynamic plastic deformation is used to consider the defomation mechanism in the above strain rate ranges, which reveals that the steep increase in the flow stress of $6061-0$ is attributed to the rate dependence of the viscous drag on the dislocation motion. It is estimated that the velocity of moving dislocation of 6061-T6 is lower than that of 6061-O at a given strain rate, and that 6061-T6 is higher in a mobile dislocation density than 6061-O. The model predicts also that the increase in the mobile dislocation density shifts the transition region, or the strain rates in which the steep increase in the flow stress becomes to appear, to the higher strain rate side
\end{abstract}

Key Words : High Strain rates, A6061, Thermally Activated Process, Viscous Drag

\section{INTRODUCTION}

It has been reported by many investigators that the strain rate dependence of the dynamic flow stress of FCC metals depends upon a instantaneous strain rate and the effect of viscous drag on the dynamic flow stress appears strongly at very high strain rates [1-4].

Recently, Sakino and Shioiri $[5,6]$ conducted a decremental strain rate tests for high-purity aluminium and copper at strain rates above about $5 \times 10^{\mathrm{J}} / \mathrm{s}$ and showed that the steep increase in the flow stress was due to a transition in the rate controlling mechanism of the dislocation motion, which shifted from the thermal activation controlled to the phonon drag controlled region. However, the interpretation for alloying metals has not yet been established because of lack of data at high strain rates above $10000 / \mathrm{sec}$ and the mechanism is still under active investigation. Yadav et al. [3] examined the effect of the strain rate on the flow stress for 6061-T6 over a very wide mange of strain rate $(0.0001$ to $100000 / \mathrm{sec})$ using a screw driven testing machine, SHPB and pressure-shear plate. They indicated the steep increase in the flow stress at the strain rate of about $200 / \mathrm{sec}$. Holt et al. [7], who studied the high strain rate mechanical behavior of $6061-O$ and $-\mathrm{T} 6$, and other aluminium alloys using SHPB, and observed very little rate sensitivity at strain rate up to $1000 / \mathrm{sec}$. Ogawa [8] performed uniaxial tensile tests on $6061-0$ and -T6 in the range of strain rate $(0.001$ to $2000 / \mathrm{sec})$ and observed no abrupt increase in the flow stress at higher strain rates. He

* Graduate school of Mechanical Engineering

* Department of Mechanical Engineering predicted also that the dynamic now stress could be represented by the thermal activation flow at a strain rate up to at least $10000 /$ sec.

In this paper, in order to clarify the strain rate dependence of the flow stress and the rate controlling mechanism of aluminium alloy, compressive tests are conducted for 6061-O and -T6 using modified SHPB in the strain rate range from about $1000 / \mathrm{s}$ to $30000 / \mathrm{s}$. Furthermore, strain rate reduction tests are made in the strain rate range above about $10000 / \mathrm{s}$. The results obtained are analysed on the basis of the dislocation kinetics using a thermal activation energy and viscous drag.

\section{EXPERIMENTAL METHOD}

\subsection{Specimens}

Measurements were made for a luminium alloys, 6061-O and -T6 (commercially available from Daido Corp.). The T-6 treatment designed by manufacturer was a solution treatment for $1 \mathrm{~h}$ at $535^{\circ} \mathrm{C}$ followed by quenching in water, and then aging for $8 \mathrm{~h}$ at a temperature of $170^{\circ} \mathrm{C}$. The $\mathrm{O}$ treatment was annealed for $3 \mathrm{~h}$ at a temperature of $380^{\circ} \mathrm{C}$ and then cooled in air. Table 1 provides the chemical composition of 6061 aluminium alloy. Specimens were machined to $1.5,2.0$ and 5.0 $\mathrm{mm}$ in both length and diameter, respectivelly from a cold-drawn bar of $6 \mathrm{~mm}$ in a diameter.

Table 1. Chemical composition of 6061 alloy ( wt \%)

\begin{tabular}{cccccccc}
\hline \hline $\mathrm{Si}$ & $\mathrm{Fe}$ & $\mathrm{Cu}$ & $\mathrm{Mn}$ & $\mathrm{Mg}$ & $\mathrm{Cr}$ & $\mathrm{Zn}$ & $\mathrm{Ti}$ \\
\hline 0.60 & 0.34 & 0.29 & 0.05 & 0.99 & 0.20 & 0.04 & 0.03 \\
\hline \hline
\end{tabular}




\subsection{Experimental Procedure}

Constant strain rate and rate reduction tests are made using the same system. In this system [9] shown in Fig. 1, a impact bar compresses directly the specimen attached at the end of a pressure bar (output bar) with silicon grease. The flow stress is measured with two semiconductor strain gauges cemented on the output bar. The signal from the strain gauges is amplified with a wide-band amplifier and stored in a high-speed digital memory (sampling time: $0.1 \mu \mathrm{s}$ ). The impact bar is made of Ti-alloy ( $\phi$ 13) and the decelerator is made of Ni-alloy (outer and inner diameter are $13 \mathrm{~mm}$ and $6 \mathrm{~mm}$, respectively). The above combination gives a reduction in strain rate by $58.6 \%$ of strain rate before reduction. The setup and specimens employed are shown in Table 2 . Using the one-dimensional bar wave approximation (elastic deformation of the impact and output bar caused by the strength of the specimen are taken into consideration), the instantaneous strain rate of the specimen is obtained in the following forms:

(a) before strain rate reduction

$$
\varepsilon=\frac{1}{\ell_{0}}\left(v_{0}-\frac{a_{0} \sigma_{0}}{A_{1} \cos _{2}}-\frac{a_{0} \sigma_{0}}{A, \cos _{3}}\right)
$$

(b) after strain rate reduction

$$
\begin{array}{r}
\varepsilon=\frac{1}{\ell_{0}}\left(\frac{v_{0}-\left(a_{0} \sigma_{0} / A_{2} c_{2} \rho_{3}\right)}{1+\left(A_{2} c_{2} \rho_{2} / A_{2} c_{3} \rho_{3}\right)}\right. \\
\left.-\frac{a_{0} \sigma_{4}}{A_{3} c_{3} \rho_{3}}\right)
\end{array}
$$

where $V_{0}$ is the initial velocity of the impact bar, $\rho, c$ and $A$ are the density, bar wave velocity and cross-sectional area, respectively; subscripts 1 and 2 correspond to the impact bar and pressure bar, respectively; $a_{0}, \ell_{0}$ and $\sigma_{n}$ are the initial cross-sectional area, initial length and instantaneous nominal flow stress of the specimen, respectively. The mass of the specimen is neglected. The true stress is calculated assuming the deformation of the specimen to be uniform.

\section{RESULTS}

Measurement of the flow stress were made for 6061-O and - T6 at strain rates from 1000 to $30000 / \mathrm{sec}$. The results are shown in Figs. 1 and 2. The data shown by symbols of $O, O$ and

Table 2. Detail of impact and output bars, decelarators and specimens employed at each strain rate range

\begin{tabular}{|l|l|c|}
\hline Strain rate range & Output bar $(\mathrm{mm})$ & Specimen $(\mathrm{mm})$ \\
\hline \hline $1000 \sim 3000 / \mathrm{s}$ & Mar. steel $\phi 20$ & $\phi 5.0, h 5.0$ \\
\hline $3000 \sim 20000 / \mathrm{s}$ & Tungsten $\phi 4$ & $\phi 2.0, h 2.0$ \\
\hline $17000 \sim 30000 / \mathrm{s}$ & Tungsten $\phi 3$ & $\phi 1.5, h 1.5$ \\
\hline
\end{tabular}

are measured using three types of output bar being different in a diameter, which correspond to $25 \mathrm{~mm}, 4 \mathrm{~mm}$ and $3 \mathrm{~mm}$ in diameter, respectively. The increase in the flow stress for 6061-0 is observed considerably at strain rate above about $10000 / \mathrm{sec}$ but its behavior is invisible in 6061-T6. It is difficult to evaluate exactly the rate dependence of the flow stress owing to scatter of the measured data. For the purpose of evaluating exactly the rate dependence of the flow stress, strain rate reduction tests are conducted at strain rates above about $10000 / \mathrm{sec}$, which is the strain rate range before reduction. Figure 3 shows a typical result of the strain rate rectuction tests for $6061-0$ at strain rate from 17500 to $7250 / \mathrm{sec}$. The strain at which the reduction of strain rate is imposed is about 0.2 . In figure, to indicate the flow stress response to sudden reduction in strain rate, the constant strain rate curve at strain rate of $17700 / \mathrm{sec}$ is also shown. Furthermore, to evaluate quantitatively the flow stress drop, an enlargement of true stress - strain curves around the rate reduction point is shown in Fig 3(b). The both curves before the rate reduction are good

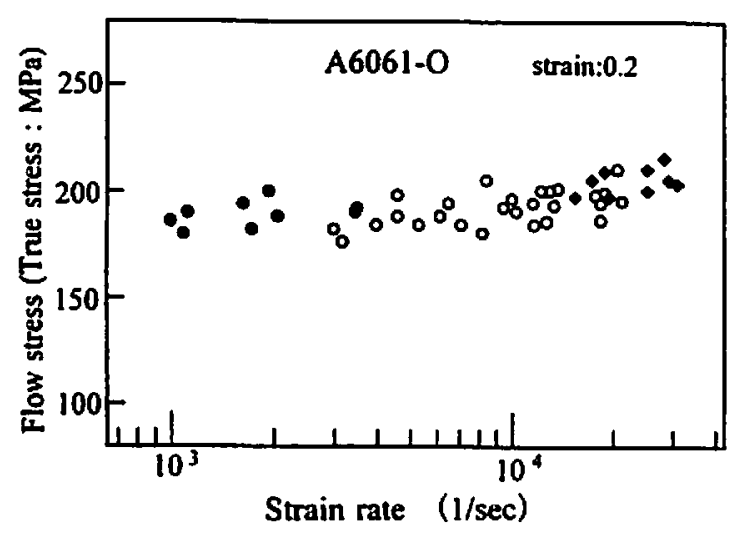

Fig. 1. Measured flow stress of $6061-0$ at strain of 0.2 in the strain rate range from $1000 \sim 30000 / \mathrm{sec}$.

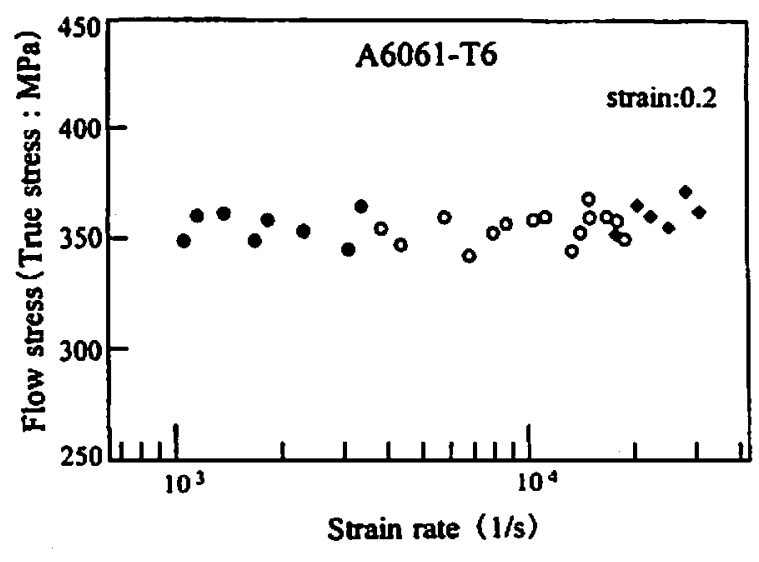

Fig. 2. Measured flow stress of 6061-T6 at strain of 0.2 in the strain rate range from $1000 \sim 30000 / \mathrm{sec}$. 


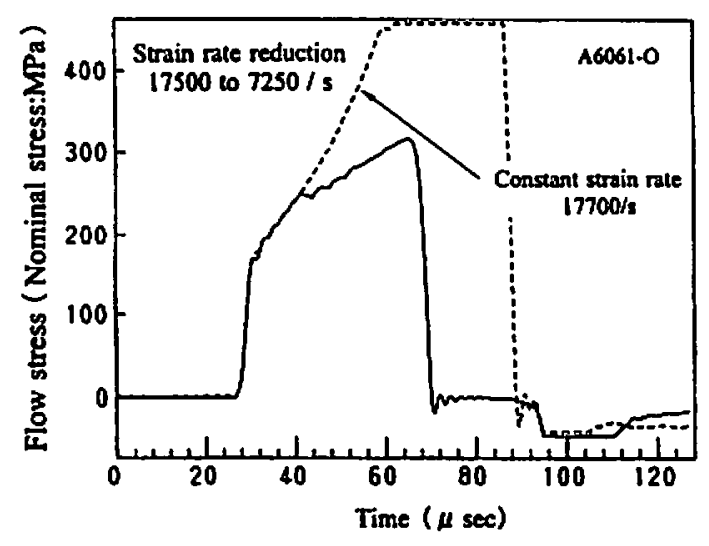

(a) Nominal stress vs. time curves

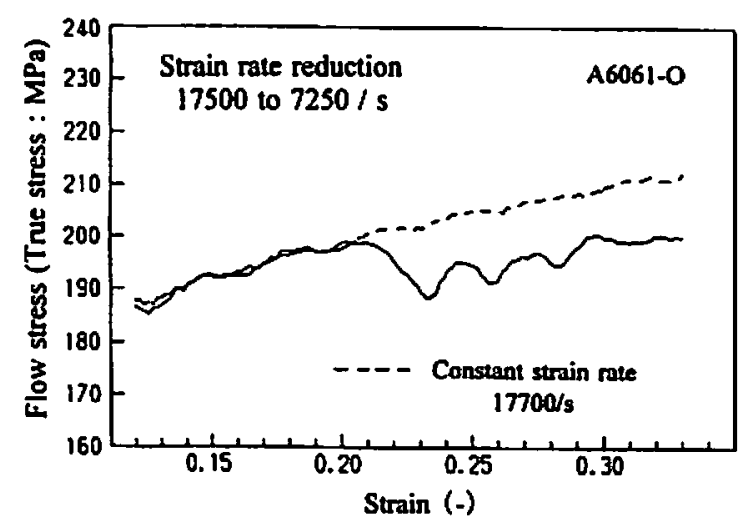

(b) True stress vs. strain curves around reduction point

Fig. 3 Response of flow stress to sudden reduction in strain rate for $6061-0$, which is from 17500 to $7250 / \mathrm{sec}$.

For evaluating the flow stress drop, flow stress at constant strain rate of $17700 / \mathrm{sec}$ is also shown.

fit. Hence, it could be considered that the flow stress drop observed is attributable to the difference in the flow stress caused by the strain rate difference. The value of the flow stress drop obtained is about $12 \mathrm{MPa}$. All results of the rate reduction tests for 6061-O are summarised in Fig.4, which represents the strain rate dependence of the flow stress which does not include an effect of a strain rate history reflecting a intemal structural evolution. It is clear that the flow stress abruptly increases at strain rate of about $5000 / \mathrm{sec}$. On the other hand, the results of strain rate reduction tests performed with 6061-T6 are shown in Figs.5 and 6, in which a reduction in strain rate is from 10800 to $6050 / \mathrm{sec}$ and 17900 to $7410 / \mathrm{sec}$, respectively. In contrast to $6061-0$, the flow stress drop caused by the rate reduction is a negligible small (that is about $3 \mathrm{MPa}$ at most in Fig.6, in which however the effect of a elastic wave dispersion becomes to be strongly) and from the above results, it should be noted that the steep increase in the flow stress does not appear at the strain rate up to at least $18000 / \mathrm{sec}$.

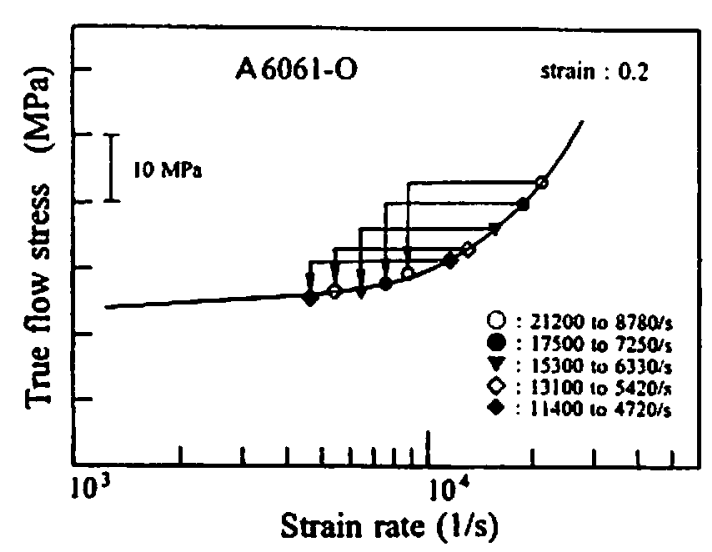

Fig. 4. Flow stress drop and instantaneous strain rate dependence of flow stress obtained from various strain rate reduction tests at strain rate ranging from about 11000 to $21000 / \mathrm{sec}$.

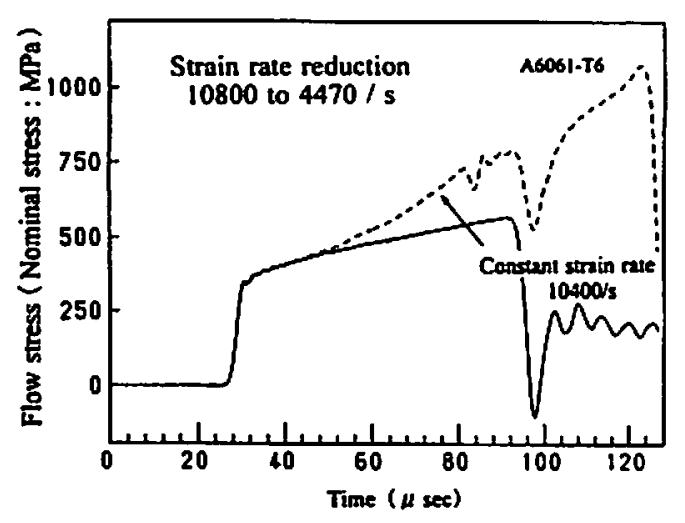

(a) Nominal stress vs. time curves

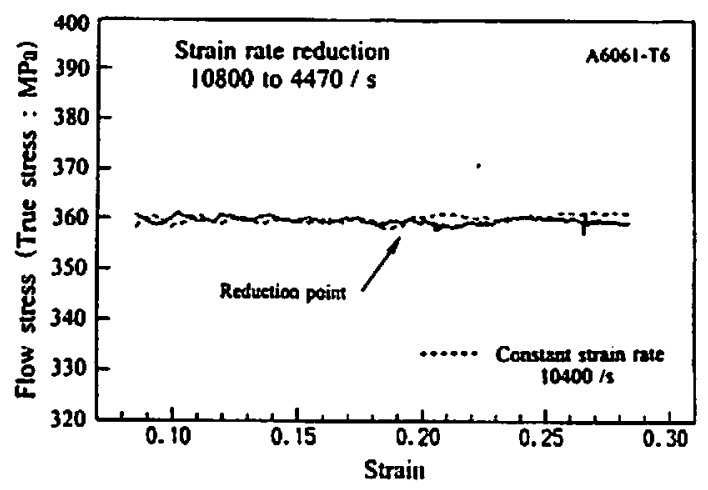

(b) True stress vs. strain curves around reduction point

Fig. 5. Response of flow stress to sudden reduction in strain rate for $6061-\mathrm{T} 6$, which is from 10800 to $4470 / \mathrm{sec}$. For evaluating the flow stress drop, fow stress at constant strain rate of $10400 / \mathrm{sec}$ is also shown. 


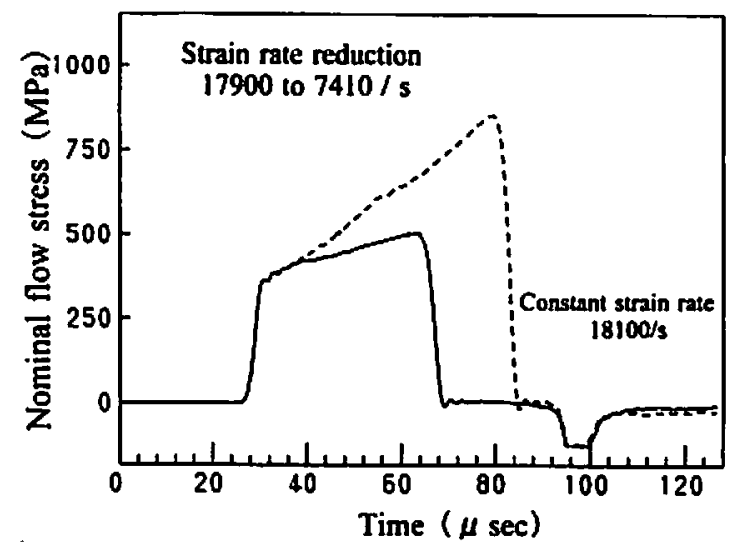

(a) Nominal stress vs. time curves

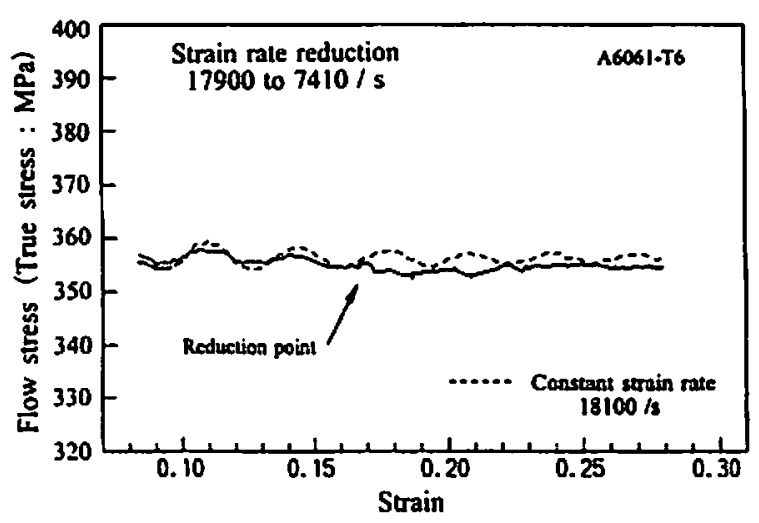

(b) True stress vs. strain curves around reduction point

Fig. 6. Response of flow stress to sudden reduction in strain rate for $6061-\mathrm{T} 6$, which is from 17900 to $7410 / \mathrm{sec}$. For evaluating the flow stress drop, flow stress at constant strain rate of $18100 / \mathrm{sec}$ is also shown.

\section{Dicussion}

By assuming that coherent precipitates as G.P. zone does not contribute explicitly to the rate sensitivity and the flow stress increasing with strain rate depends primarily on the instantaneous strain rate, a analysis is made on the basis of the dislocation kinetics $[1,4,10]$. Usually in FCC metals, the mobile dislocation segments move repeating the thermally assisted cutting of the forest dislocations and the phonon drag controlled jump motion alternately. By simplifying the unit motion of the dislocation segment, the rate of the shear strain can be given by the following equation:

$$
\dot{\gamma}=\frac{N L^{2} b}{t_{1}+t_{v}}
$$

$$
\begin{gathered}
=\frac{\frac{N L^{2} b}{v^{-1} \exp \left(-\left[U-L b^{2}(\tau-\tau a) J / k T\right\}\right.}}{\frac{+L B /(\tau-\tau a) b}{}}
\end{gathered}
$$

where $t_{1}$ is the waiting time for the thermally assisted cutting, $t \boldsymbol{r}$ is the time required by one jump motions under the control of the phonon drag, $N$ is the number of the moving segments per unit volume, $L$ is the distance between the adjacent forest dislocations, $b$ is the Burgers vector, $U$ is the activation energy of cutting, $v$ is the frequency factor, $k$ is the Boltzmann constant, $T$ is the absolute temperature, $\tau$ is the resolved shear stress, $\tau_{a}$ is the long range athermal stress, and $B$ is the phonon drag coefficient. At lower strain rates (i.e. at lower stresses) $t, \gg t \vee$ and Eq. (3) can be approximated as

$$
\dot{\gamma}=N L^{2} b v \exp \left\{-\left[U-L b^{2}(\tau-\tau a)\right] / k T\right\}
$$

This is the thermal activation flow rate-controlled by the thermally assisted cutting of the forest dislocations, in which the flow stress depends linearly upon the logarithm of the strain rate. At very high strain rates(i.e. at higher stresses) $t, \ll t v$ and Eq. (3) is approximated as

$$
\dot{\gamma}=N L^{2} b(\tau-\tau a) / B
$$

This is the phonon drag controlled viscous flow in which the flow stress depends linearly upon the strain rate itself. Equation (3) can cover a wide strain rate range including thermal activation flow range and the phonon drag controlled flow range together with the transition range between them.

The unknown quantities in $\mathrm{Eq}$. (3) are the mean distance between the adjacent forest dislocations, $L$, the density of moving dislocations, $N L$, and the long range athermal stress, $\tau$ a. Those quantities will depend upon the structure formed during the deformation, and in the present treatment the values of those quantities are determined so that a good fit of the predicted curves to the experimental data is obtained If $L, N L$ and $\tau$ a are independent of the strain rate, $L$ is determined from the gradient of the $\sigma$ vs. $\log \varepsilon$ plot of the experimental flow stress in a relatively low strain rate range where $\sigma$ is linearly dependent upon $\log \varepsilon . N L$ is determined from the strain rate at which the flow stress begins to rise steeply, and $\tau_{a}$ is determined to obtained a good fit in the stress level. Thus determined values of $L, N L$ and $\tau_{0}$ for $6061-0$ are $6.71 \times 10^{-8} \mathrm{~m}, 5.51 \times 10^{11} \mathrm{~m}^{-2}$ and 47.7 $\mathrm{MPa}$, respectively. The Taylor factor is assumed to be 3.07 .

The curve predicted by Eq. (3) using the above numerical values is shown by a dot-dash line in Fig. 7 together with the experimental flow stress and the solid line obtained in Fig. 4. In the calculation, it is assumed that $N L, L$ and $\tau$ a are independent of the strain rate. The steep rise seen in the predicted curve is due to the transition from the thermal activation flow to the phonon 
drag controlled flow. Therefore, it can be considered that the steep rise in the flow stress observed in the experimental data is caused by the phonon drag upon dislocation motion.

However, the degree of the steep increase observed in the measured flow stress is relatively low in comparison with that predicted by Eq. (3). This difference is interpreted as follows: in the model the moving dislocation segments hung between adjacent forest dislocations have the same length, and when the strain rate (accordingly flow stress) becomes high all of the segments shift simultaneously to the viscous drag-controlled state, while in the real materials, since the array of the forest dislocations should be random and accordingly the length of the segments should have a statistical distribution, such the simultaneous transition will not occur.

In this investigation, of particular interest is the observation that the same behavior as that appears in 6061-O is not observed for 6061-T6. The explanation may be allowed that the velocity of the moving dislocation does not attain the critical one at which the effect of phonon drag on the movement of the dislocation becomes dominant. That is to say, the mean dislocation velocity of 6061-T6 is lower than that of 6061-O at fixed strain rate. If so, it is considered from Eq.(3) that 6061-T6 is higher in the moving dislocation density than 6061-0.

Figure 8 shows the flow stress-strain rate relations calculated by taking the moving dislocation density as a parameter. The activation volume $L b^{2}$ and the phonon drag coefficient $B$ are independent of the moving dislocation density. It is clear that the increase in the moving dislocation density shifts the transition region, or the strain rate in which the steep increase in the flow stress appears, to the higher strain rate side.

\section{CONCLUSION}

The high strain rate tests were performod with aluminium alloys, 6061.0 and - T6 in the strain rate range from about $1000-30000 / \mathrm{sec}$. For $6061-0$, the steep increase in the flow stress was observed at strain rate of about 5000/sec, but for 6061-T6, the behavior was not observed at strain rates up to about $20000 / \mathrm{sec}$. By analyzing quantitatively the obtained results on the basis of the dislocation kinetics, it is confirmed that the steep increase in the flow stress observed for $6061-0$ at high strain rates is attributed to the transition in the rate controlling mechanism of the dislocation motion from the thermal activation controlled flow to the phonon drag controlled flow. Furthermore, it is supposed that the increase in the mobile dislocation density lowers the dislocation velocity at a given strain rate and as a result, shifts the above mentioned transition range to the higher strain rate side.

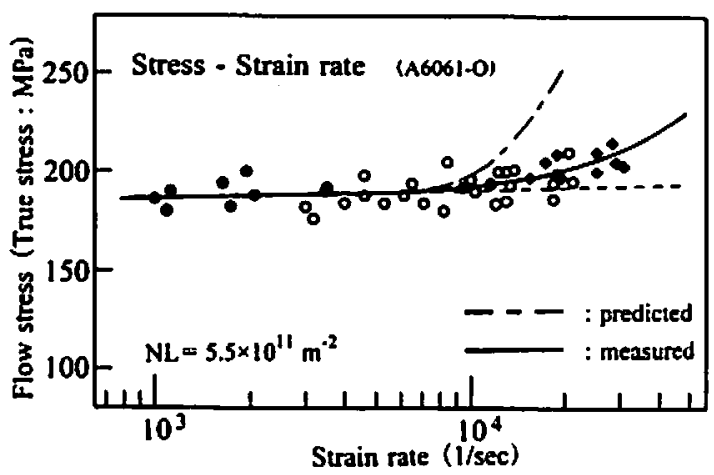

Fig. 7. Comparison of measured flow stress with the prediction (dot-dash line) determined from Eq. (3).

Solid line is taken from Fig.4.

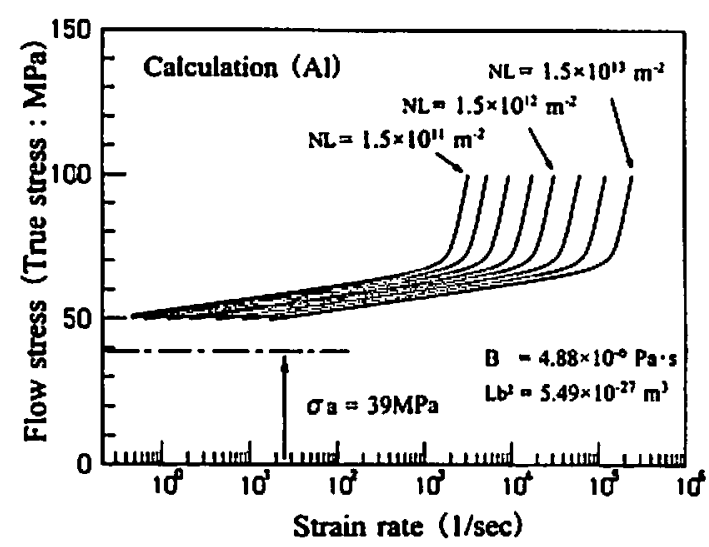

Fig. 8. Relation between strain rate at which steep increase in flow stress appears and moving dislocation density.

\section{REFERENCES}

[1] W. G. Ferguson, A. Kumar and J. E. Dom, J. Appl. Phys., 38 (1967), P.1863.

[2] R. J. Clifton, J. Appl. Mechanics (Tras. ASME), 50 (1983), P.941.

[3] S.Yadav, D. R. Chichili and K. T. Ramesh, Acta Metall. Mat, 43 (1995), P.4453.

[4] J. Shioiri, K. Sakino and S. Santoh, Proc. IUTAM Symp. Consstituteve Relation in High/Very High Strain Rates, Eds. K. Kawata and J. Shioiri, Springer-Verlag Tokyo (1997), P.49.

[5] K. Sakino and J. Shioiri, Trans. Jpn. Soc. Mech. Eng., Inter. Joumal, 39 (1996), P.135.

[6] K. Sakino, Joumal de Physique III, (2000), P.9.

[7] D. L. Holt, S. G. Babcok, S. J. Green and C. J. Maiden, Trans. ASM, 60 (1967), P.119.

[8] K. Ogawa, Joumal JILM, $51(2001)$, P. 175. (in Japanese)

[9] K. Sakino and J. Shioiri, Joumal de Physique III, Vol.1, octobre (1991), C3-35.

[10] A. Kumar and R.G. Kumble , Appl. Phy., 40 (1969), P.3475.

[11] M. F. A shby and H. J. Frost, Ed. A. S. Argon, The MTT Press, Cambridge (Massachusetts) \& London (England) (1975), PII7.

[12] A. Hikata, R. A. Johnson and C. Elbaum, Physical Review B, 2 (1970), P.4856. 